\title{
Degradable/non-degradable polymer composites for in-situ tissue engineering small diameter vascular prosthesis application
}

\author{
Fujun Wang ${ }^{\mathrm{a}, \mathrm{b}}$ Abedalwafa Mohammed ${ }^{\mathrm{a}, \mathrm{b}}$ Chaojing $\mathrm{Li}^{\mathrm{a}, \mathrm{b}}$ Peng Ge $^{\mathrm{a}, \mathrm{b}}$ Lu Wang $^{\mathrm{a}, \mathrm{b}}$ and Mar- \\ tin W. King a, b, c \\ ${ }^{a}$ Key Laboratory of Textile Science and Technology, Ministry of Education, Donghua University, \\ Shanghai 201620, China \\ ${ }^{b}$ College of Textiles, Donghua University, Shanghai 201620, China \\ ${ }^{c}$ Department of Textile Engineering, Chemistry \& Science, North Carolina State University, \\ Raleigh, NC, USA
}

\begin{abstract}
Various tissue-engineered vascular grafts have been studied in order to overcome the clinical disadvantages associated with conventional prostheses. However, previous tissue-engineered vascular grafts have possessed insufficient mechanical properties and thus have generally required either preoperative cellular manipulation or the use of bioreactors to improve their performance. In this study, we focused on the concept of in situ cellularization and developed a tissueengineered vascular graft with degradable/ non-degradable polymer composites for arterial reconstruction that would facilitate the renewal of autologous tissue without any pretreatment. Additionally, these composites are designed to improve the mechanical performance of a small-diameter vascular prosthesis scaffold that is made from a flexible membrane of poly $(\varepsilon-$ caprolactone) (PCL). The PCL scaffold was reinforced by embedding a tubular fabric that was knitted from polyethylene terephthalate (PET) yarns within the freeze-dried composite structure. Adding this knitted fabric component significantly improved the mechanical properties of the composite scaffold, such as its tensile strength and initial modulus, radial compliance, compression recovery, and suture retention force. Finally, this reinforced composite structure is a promising candidate for use as a tissue-engineered scaffold for a future small diameter vascular prosthesis.
\end{abstract}

Keywords: Vascular prosthesis, knitted fabric, poly ( $\varepsilon$-caprolactone), composite structure, mechanical properties, reinforcement

\footnotetext{
${ }^{*}$ Corresponding author: Lu Wang, Key Laboratory of Textile Science and Technology of Ministry of Education, College of Textiles, Donghua University, Shanghai, China. Tel.: 086-21-67792637; Fax: 086-21-67792637; E-mail: wanglu@dhu.edu.cn.
}

0959-2989/14/\$27.50 @ 2014 - IOS Press and the authors. 


\section{Introduction}

Atherosclerotic vascular disease is pathological condition characterized by the hardening, narrowing, and occlusion of arteries and may therefore lead to numerous adverse health conditions: claudication pain, numbness, gangrene, as well as the possibility of amputation. In response, a variety of tissueengineering therapies have been investigated with the ultimate objective of developing a functional small-diameter vascular prosthesis [1-4]. Indeed, these tissue-engineered vascular grafts (TEVG) are considered to be potentially advantageous over the current use of the patients' autologous tissue, such as the saphenous vein [5-7]. However, both the biodegradable scaffold's structural design, as well as its material components, remain critical aspects in the engineering of a functional and biocompatible vascular substitute [8-10]. Furthermore, numerous limitations still remain despite the many ongoing efforts to develop such scaffolds [11,12]. For example, the long-term patency of a TEVG, or the time span for which blood will continue to flow, is influenced by the structure's biomechanics [13]. As the TEVG is constantly subjected to dynamic shear stresses, wall tensile, and radial stresses from the pulsatile blood flow, the structure should have sufficient ultimate bursting strength. Concurrently, for surgeons to confidently perform anastomoses, adequate suture retention strength is also crucial, and in addition, the device needs radial compliance to avoid intimal hyperplasia [14].

A natural artery structure contains a series of layers, with each layer being responsible for one aspect of the whole vessel's mechanical behavior $[11,13,15]$. Therefore, we decided to fabricate a smalldiameter tubular scaffold and then reinforce it by adding a middle knitted tubular layer in order to improve its mechanical performance. In this study, PCL was selected as the scaffold material for several reasons: namely its biocompatibility, compliance and extensibility, and appropriate bioresorption profile. Additionally, it has been approved by the Food and Drug Administration (FDA) as a suture material and has also been widely used in various tissue engineering applications. For the reinforcing knitted component, PET yarns were chosen because of their long clinical history in fabricating vascular prosthesis.

\section{Experimental}

\subsection{Materials}

The PET multifilament yarn (50 denier, 36 filaments) that was used in this study was supplied by the Fangxin Chemical Fiber Company (Shaoxing, China). The PCL polymer ( $\mathrm{Mw}=80,000 \mathrm{~g} / \mathrm{mol}$ ) was purchased from the Brightchina Industrial Co., Ltd., Shenzhen, China, and the analytical grade acetic acid was obtained from the Sinopharm Chemical Reagent Co., Ltd., Shanghai, China.

\subsection{Preparation of a reinforced PCL composite scaffold}

A PCL tubular freeze-dried scaffold was fabricated by dissolving PCL in acetic acid and then preparing a $15 \mathrm{wt} \%$ solution. Next, this solution was coated on to the outside of a $6 \mathrm{~mm}$ diameter polytetrafluorothylene (PTFE) rod that served as the mold to control the inside diameter of the tubular scaffold. Furthermore, additional layers of coating were added to control the wall thickness to within $0.20 \pm 0.02 \mathrm{~mm}$. Then, to reinforce this scaffold, PET yarns were knitted into a $6 \mathrm{~mm}$ diameter single jersey-knitted structure using a 25 needle circular weft knitted machine that was specially designed and built in our laboratories. The PCL solution was then coated on to both sides of this tubular knitted 
structure prior to mounting it on the $6 \mathrm{~mm}$ diameter PTFE rod. After coating, the PCL composite scaffolds were cooled and then held at $-10^{\circ} \mathrm{C}$ for $12 \mathrm{~h}$. They were then freeze-dried at $-60^{\circ} \mathrm{C}$ at a pressure of $10 \mathrm{~Pa}$ of less for $5 \mathrm{~h}$ in a model FD-1A-50 freeze drying machine. The scaffolds were subsequently immersed into a series of 3 distilled water baths; each bath was for a $12 \mathrm{~h}$ period so as to remove any residual solvent.

\subsection{Characterization methods}

The scaffold morphology was examined by a Hitachi scanning electron microscope (Model "S4800"). Additionally, the scaffolds' tensile strength and initial modulus were measured in both the longitudinal and circumferential direction on a Wenzhou Darong (Model YG-B026H) universal mechanical tester. The strain rate was maintained at $50 \mathrm{~mm} / \mathrm{min}$, and the gauge length for the longitudinal and circumferential test was $60 \mathrm{~mm}$ and $18.8 \mathrm{~mm}$, respectively. Each sample was tested in triplicate, and the values were calculated and reported as the mean \pm standard deviation (SD) in Table 1.

The suture retention force was tested according to Standard ISO 7198: 1998 [16]. Furthermore, the scaffold's elastic recovery after compression was determined on a Laizhou Electronic Instrument (Model YG061) radial compression apparatus. The compression rate was $10 \mathrm{~mm} / \mathrm{min}$, and the samples were compressed to $50 \%$ of the original diameter using a $3 \mathrm{~mm}$ diameter indenter. The compression recovery of each sample was calculated according to the following Eq. (1):

$$
E \%=\frac{E_{1}+E_{2}}{E_{T}} \times 100 \%
$$

where E1 is the delayed elastic deformation as measured after 10 seconds, E2 is the spontaneous elastic deformation as measured at the moment of removing the external force, and ET is the total deformation.

The tubular dynamic radial compliance was determined by measuring the percentage change in the internal radius with changes in the internal pressure, as presented in Eq. (2) [17]. Additionally, the measurements of the pressurized external diameter were taken at different pressures using a dynamicpulsatile system (Bose Corporation, MN, USA). The measured external diameters were then converted to pressurized internal radii according to Eq. (3):

$$
\begin{aligned}
& C \%=\frac{\left(R_{p 2}-R_{p 1}\right) \times 10^{4}}{R_{p 1} \times\left(P_{2}-P_{1}\right)} \\
& R_{p}=\frac{D_{p}}{2}-t
\end{aligned}
$$

where $\mathrm{C} \%$ is the radial compliance, $\mathrm{Rp}$ is the internal radius $(\mathrm{mm})$ corresponding to pressure $\mathrm{P}$ $(\mathrm{mmHg})$, whereas $\mathrm{Dp}$ is the measured external diameter $(\mathrm{mm})$ corresponding to pressure $\mathrm{P}(\mathrm{mmHg})$, and $t$ is the graft wall thickness $(\mathrm{mm})$. 
The data was statistically analyzed by a student t-test to determine whether differences in the mechanical properties with and without reinforcement were significant at the $95 \%$ confidence interval $(\mathrm{p} \leq$ $0.05)$.

\section{Results and discussion}

Images of the reinforced PCL/PET freeze-dried composite scaffold are shown in Figure 1. Figure 1(a) presents a schematic diagram that reveals the three layer structure of the reinforced PCL/PET composite scaffold. The illustration indicates that the reinforcing weft-knitted tubular structure was embedded between two layers of the PCL porous matrix. Figure 1(b) illustrates the single jersey weftknitted structure composed of PET multifilament yarns. Once fabricated and freeze-dried, the circular PCL scaffold had a $0.2 \mathrm{~mm}$-thick wall (Figure 1(c)). The SEM image of the composite scaffold shows that the solidified membrane had numerous $1 \sim 2 \mu \mathrm{m}$ in diameter pores that resulted from phase separation during the freeze-drying (Figure 1(d)) [18]. Its appearance was similar to that of a commercial ePTFE vascular graft as shown in Figure 1(e). The SEM micrograph of the reinforced composite scaffold confirms that the PET fibers were intimately embedded within the PCL matrix and therefore were able to improve the scaffold's mechanical performance (Figure 1(f)). These observations confirm that adding a knitted fabric layer successfully reinforced the freeze-dried resorbable PCL scaffold and, in the process, created a microporous morphology with a similar appearance to that of a commercial ePTFE vascular graft.
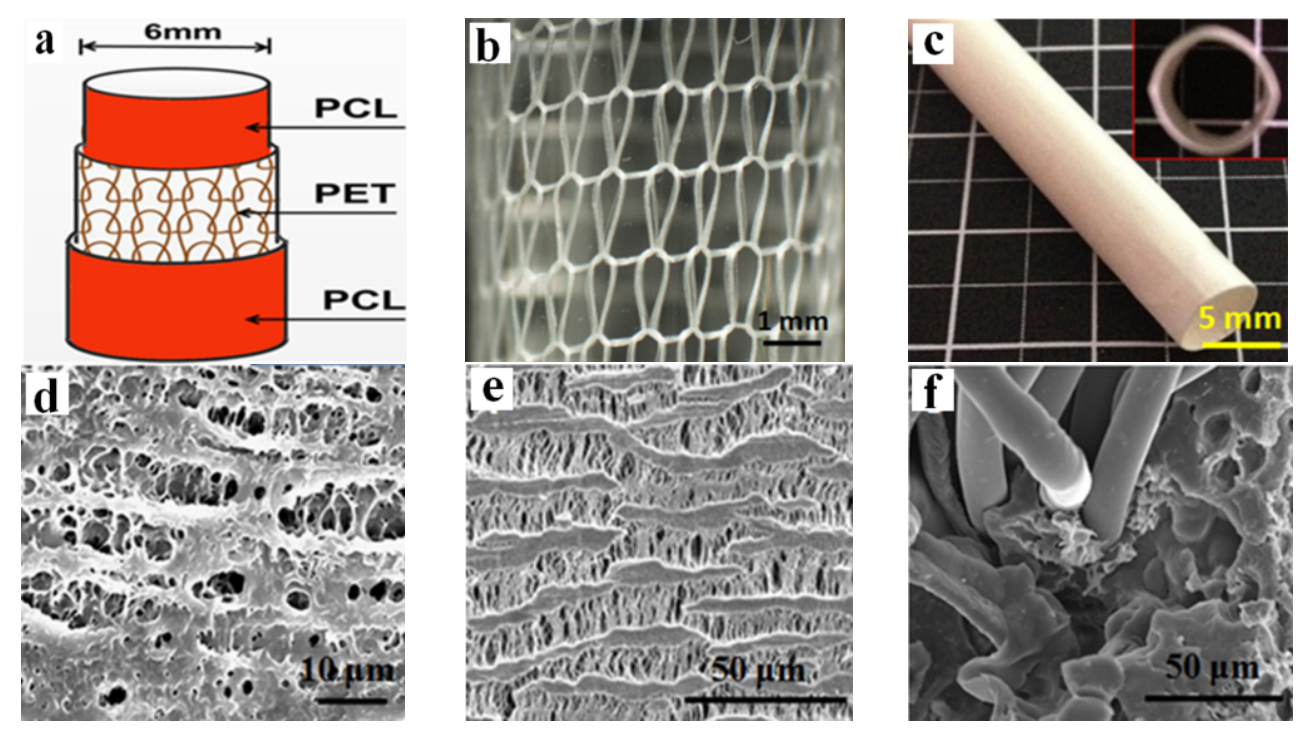

Fig. 1. (a) Schematic diagram representing the three layered structure of the reinforced PCL/PET composite scaffold. (b) Optical image of the PET jersey knit structure and (c) PCL/PET freeze-dried composite scaffold. (d) SEM image showing the porous microfibrillar morphology of the PCL/PET composite scaffold, which has a similar appearance to the (e) SEM image of a commercial ePTFE vascular graft. (f) SEM photomicrograph showing reinforcing fiber/PCL matrix bonding at the fiber/matrix interface following delamination in a cryobending test. 
Table 1

The mechanical properties of the unreinforced and reinforced PCL scaffolds

\begin{tabular}{lll}
\hline & Pure PCL scaffold & PCL/PETcomposite scaffold \\
\hline Circumferential tensile strength (MPa) & $7.35 \pm 0.17^{*}$ & $10.37 \pm 1.00^{*}$ \\
\hline Circumferential initial modulus (Mpa) & $2.39 \pm 0.03$ & $2.24 \pm 0.04$ \\
\hline Longitudinal tensile strength (MPa) & $4.93 \pm 0.37^{*}$ & $12.11 \pm 0.54^{*}$ \\
\hline Longitudinal initial modulus (Mpa) & $4.89 \pm 0.34^{*}$ & $5.47 \pm 0.80^{*}$ \\
\hline Compression force $(50 \%)(\mathrm{cN})$ & 79.23 & 123.34 \\
\hline Compression recovery (\%) & 89.9 & 93.85 \\
\hline
\end{tabular}

Note: *Statistical analysis was performed using a t-test to compare the data with and without reinforcement, and the probability value of $p \leq 0.05$ was considered to indicate a statistically significant difference.

As detailed in Table 1, the unreinforced PCL scaffolds' mechanical properties significantly increased because of the addition of the knitted reinforcing layer. Furthermore, the longitudinal initial modulus grew from 4.89 to $5.47 \mathrm{MPa}$, while the tensile strength climbed from 4.93 to $12.11 \mathrm{MPa}$. In terms of the circumferential initial modulus and tensile strength, the values presented in Table 1 suggest a similar enhancement due to the reinforcing layer addition. However, statistical analysis has not confirmed that such improvements are significant at the $95 \%$ confidence interval. Additionally, the extent of compression recovery for the reinforced PCL/PET composite scaffold increased from $89.9 \%$ to $93.85 \%$. This demonstrates that the scaffold will more easily resume its original dimensions as well as be easier to maintain and restore its original shape following compression loading, flexing, and/or kinking.

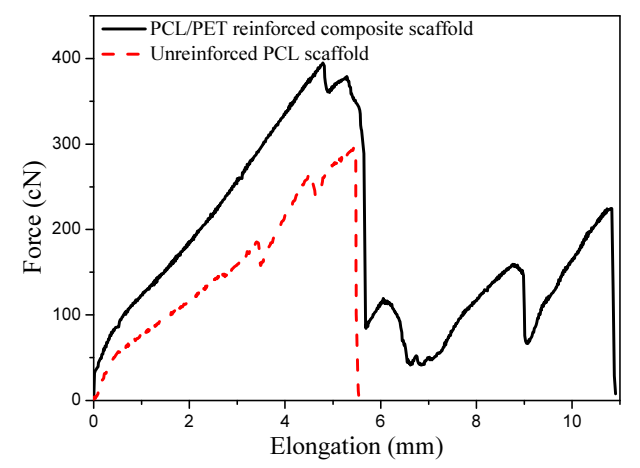

Fig. 2. Typical suture retention force elongation curves for the reinforced and unreinforced PCL scaffolds.

Table 2

Comparison of dynamic radial compliance of the unreinforced and reinforced PCL scaffolds with typical commercial vascular grafts and a small caliber natural artery

\begin{tabular}{lllll}
\hline No. & Samples & Thickness $(\mathrm{mm})$ & Radial Compliance $(\% / 100 \mathrm{mmHg})$ & Ref. \\
\hline 1 & Reinforced PCL & $0.193 \pm 0.023$ & 1.179 & This study \\
\hline 2 & Unreinforced PCL & $0.182 \pm 0.058$ & 1.188 & This study \\
\hline 3 & ePTFE & 0.600 & $0.31-0.57$ & {$[13]$} \\
\hline 4 & Woven & 0.100 & $0.57-0.81$ & {$[13]$} \\
\hline 5 & Knitted & 0.35 & $0.94-2.52$ & {$[13]$} \\
\hline 6 & Porcine carotid & - & $7.21-10.04$ & {$[13]$} \\
\hline
\end{tabular}


Figure 2 shows the typical suture retention force-elongation curves for the unreinforced and reinforced PCL scaffolds. From these curves, it can be seen that as a result of adding the knitted component, the maximum force increases, the elongation at break is extended, and the scaffold's toughness is enlarged by increasing the total area (energy) under the curve. As was mentioned in the introduction, small caliber vascular prostheses need superior radial compliance so as to avoid complications, such as intimal hyperplasia, that are caused by a compliance mismatch at the distal anastomosis [19]. Table 2 provides the average dynamic radical compliance values as measured for the unreinforced and the reinforced PCL scaffolds that were prepared in this study. The results reveal that the radial compliance significantly improved by adding a knitted reinforcing layer as compared to the woven and microporous ePTFE fluorocarbon vascular prostheses. However, when these radial compliance values are compared with those for natural arteries, it becomes evident that further work is necessary in order to improve the compliance of these artificial scaffolds so that they resemble natural vessels.

\section{Conclusion}

The fabrication of PCL scaffolds for small diameter blood vessel replacements has been successfully demonstrated by adding a reinforcing knitted fabric layer into a freeze-dried tubular PCL membrane. The reinforced scaffolds' mechanical properties, such as their tensile strength, initial modulus, compression recovery, and suture retention force, were significantly improved compared to those of the unreinforced sample. Meanwhile, both the unreinforced and the reinforced scaffolds exhibited comparable radial compliance. Furthermore, the SEM micrographs exhibit a microporous morphology similar to that of commercial ePTFE vascular prostheses. Next, this reinforced scaffold will be evaluated in an animal model in order to determine its suitability as a template for a small caliber vascular graft.

\section{Acknowledgement}

This work was supported by the Fundamental Research Funds from the Central Universities (NS2013), the National Natural Science Foundation of China (31100682) and the Seed Fund from the Engineering Research Center of Technical Textiles, Ministry of Education, China.

\section{References}

[1] J.P. Stegemann, S.N. Kaszuba and S.L. Rowe, Review: Advances in vascular tissue engineering using protein-based biomaterials, Tissue. Eng. 13 (2007), 2601-2613.

[2] W. Zhang, W. Liu, L. Cui and Y. Cao, Tissue engineering of blood vessel, J. Cell. Mol. Med. 11 (2007), 945-957.

[3] H. Naderi, M.M. Matin and A.R. Bahrami, Critical issues in tissue engineering: biomaterials, cell sources, angiogenesis, and drug delivery systems, J. Biomater. Appl. 26 (2011), 383-417.

[4] D. Lamprou, P. Zhdan, F. Labeed and C. Lekakou, Gelatine and gelatine/elastin nanocomposites for vascular grafts: processing and characterization, J. Biomater. Appl. 26 (2011), 209-215.

[5] R.M. Nerem and D. Seliktar, Vascular tissue engineering, Ann. Rev. Biomed. Eng. 3 (2001), 225-243.

[6] O.E. Teebken and A. Haverich, Tissue engineering of small diameter vascular grafts, Eur. J. Vasc. Endovasc. Surg. 23 (2002), 475-485.

[7] J. Hu, W. Chao, P. Lee and C. Huang, Construction and characterization of an electrospun tubular scaffold for smalldiameter tissue-engineered vascular grafts: A scaffold membrane approach, J. Mech. Behav. Biomed. Mater. 13 (2012), $140-155$

[8] P. Ma, Biomimetic materials for tissue engineering, Adv. Drug. Del. Rev. 60 (2008), 184-198. 
[9] Y. Wang, D.E. Noga, K. Yoon, A.M. Wojtowicz, A. Lin and A.J. Garcia, Highly porous cross-linkable PLA-PNB block copolymer scaffolds, Adv. Funct. Mater. 18 (2008), 3638-3644.

[10] H. Ma, J. Hu and P. Ma, Polymer scaffolds for small-diameter vascular tissue engineering, Adv. Funct. Mater. 20 (2010), 2833-2841.

[11] R.M. Nerem, Tissue engineering a blood vessel substitute: the role of biomechanics, Yonsei Med J. 41 (2000), 735-739.

[12] S. Greenwald and C. Berry, Improving vascular grafts: the importance of mechanical and haemodynamic properties, J. Pathol. 190 (2000), 292-299.

[13] D.R. Nisbet, J.S. Forsythe, W. Shen, D.I. Finkelstein and M.K. Horne, A review of the cellular response on electrospun nanofibers for tissue engineering, J. Biomater. Appl. 24 (2009), 7-29.

[14] Y. Mine, H. Mitsui, Y. Oshima, Y. Noishiki, M. Nakai and S. Sano, Suture retention strength of expanded polytetrafluoroethylene (ePTFE) graft, Acta. Med. Okayama 64 (2010), 121-121.

[15] H.J. Salacinski, S. Goldner, A. Giudiceandrea, G. Hamilton, A.M. Seifalian, A. Edwards and R.J. Carson, The mechanical behavior of vascular grafts: A review, J. Biomater. Appl. 15 (2001), 241-278.

[16] G. Shen, J. Lin, H. Zhang and L. Wang, Comparison of the radial compliance of three kinds of vascular prostheses and pig's carotid based on dynamic-simulated condition, Proc. Worl. Cong. Medic. Phys. Biomed. Eng. Chin., 2013, 176179.

[17] R. Tu, J. Macintyre, C. Hata, C.L. Lu, E. Wang and R.C. Quijano, Dynamic internal compliance of a vascular prosthesis, ASAIO Trans. 37 (1991), 470-472.

[18] Y. Minaberry and M. Jobbágy, Macroporous bioglass scaffolds prepared by coupling sol-gel with freeze drying, Chem. Mater. 23 (2011), 2327-2332.

[19] P.M. Crapo and Y. Wang, Physiologic compliance in engineered small-diameter arterial constructs based on an elastomeric substrate, Biomater. 31 (2010), 1626-1635. 